\title{
Original
}

\section{The Association between Changes in Smoking Status and Cardiovascular Disease Risk Factors from Childhood to Young Adulthood in Longitudinal Cross-sectional Cohorts; The Bogalusa Heart Study}

\author{
Hiroto Araki ${ }^{1)}$, Jeanette Gustat ${ }^{2)}$, Takeshi KawaguchI ${ }^{1}{ }^{1}$ \\ and Gerald. S. BERENSON ${ }^{2)}$
}

\begin{abstract}
The aim of this study was to examine the effects of smoking on changes in cardiovascular disease risk factors between childhood and young adulthood. Blood pressure, serum lipoproteins and body mass index were measured in 2258 individuals; as children in 4 baseline surveys (1974-1977) and as young adults in 3 follow-up surveys (1985-1996), in the biracial community of Bogalusa, Louisiana. Smoking variables were defined as categories based on (1) present smoking status, (2) cumulative smoking volume (packyears ever smoked), and (3) years since quitting smoking. We found that in heavier smokers there was a significantly greater increase in low-density lipoprotein cholesterol and serum triglycerides, and a decrease in high-density lipoprotein cholesterol. These changes showed appropriate dose-response gradients. The length of time individuals remained smoke-free was related to significantly smaller increases in blood pressure and serum triglycerides (in both sexes), and to an increase in body mass index in males only. These findings support the use of pack-years as an appropriate indicator for evaluation of changes in risk factor variables. The results suggest that even in young adulthood, smoking causes adverse changes in the risk factors for cardiovascular disease, and that quitting smoking earlier brings about beneficial changes.
\end{abstract}

Key words : young adulthood, cross-sectional, smoking, cardiovascular diseases risk factor, pack-years

\section{Introduction}

Tobacco use is an obvious risk factor for a variety of diseases. Worldwide, the proportions of disability-adjusted life years (DALYs) attributed to smoking are $66 \%$ for lung cancer, $38 \%$ for chronic obstructive pulmonary disease, $16 \%$ for mouth and oropharynx cancers, and $12 \%$ for cardiovascular disease $(\mathrm{CVD})^{1)}$. In developed countries like the United States, the smoking-attributable fractions of DALYs are $90 \%$ (male) and $69 \%$ (female) for lung cancers, $79 \%$ (male) and 57\% (female) for chronic obstructive pulmonary disease,

1) Department of Public Health, Showa University School of Medicine, 1-5-8 Hatanodai, Shinagawa-ku, Tokyo 1428555 , Japan.

2) Tulane Center for Cardiovascular Health and Department of Epidemiology, Tulane School of Public Health and Tropical Medicine, Tulane University Health Sciences Center. 
and $32 \%$ (male) and $10 \%$ (female) for $\mathrm{CVD}^{1)}$. Smoking has negative effects on CVD risk factors in adulthood, with a consequent impact on mortality rate ${ }^{2)}$. Smoking, together with various other risk factors related to life-style, including hypertension, high cholesterol and obesity, has a large influence on overall mortality. For example, in developed countries in the North and South Americas, the proportions of mortality attributable to smoking are $33 \%$ for males and $30 \%$ for females ${ }^{3)}$. In comparison, high blood pressure accounts for $17 \%$ of mortality in males and $19 \%$ in females, cholesterol disorders $15 \%$ in males and $19 \%$ in females, and being overweight $13 \%$ in males and $14 \%$ in females ${ }^{3)}$.

In the United States, heart disease has been the leading cause of death in both sexes for several decades ${ }^{4)}$. Cigarette smoking is the most important modifiable risk factor for heart disease in adults ${ }^{5-7)}$, as it is for pulmonary disease and various cancers. In addition, smoking produces substantial health-related economic costs to society ${ }^{8)}$. In 2000 , the most common causes of death in the United States were related to tobacco, poor diet and physical inactivity ${ }^{9.10)}$.

Tobacco smoke, high blood cholesterol, high blood pressure, physical inactivity, obesity and diabetes are the 6 major independent risk factors for coronary heart disease that we can modify or control ${ }^{7)}$. Smoking leads to a higher risk of developing a number of chronic disorders such as fatty build-up in arteries, several types of cancer, and chronic obstructive pulmonary disease. Smoking is associated with increased blood pressure, elevated serum triglycerides (TG) and low-density lipoprotein cholesterol (LDL-C), and decreased high-density lipoprotein cholesterol (HDL-C) ${ }^{11,12)}$. Increasing cumulative smoking volume (measured in pack-years) induces higher risk development for CVD, while those who quit smoking have a reduced or reversed risk compared with those who continue to smoke ${ }^{13-15)}$. Pack-years of smoking (but not current smoking status) is associated with progression of atherosclerosis, so some adverse effects of smoking for CVD may be cumulative and irreversible ${ }^{15)}$.

Our objective was to examine the association between smoking (as measured by current status, pack-years, and years since quitting) and changes in CVD risk factors over time, in adolescents and young adults. To achieve this we used multiple cross-sectional cohorts of the Bogalusa Heart Study.

\section{Methods}

\section{Population}

The Bogalusa Heart Study is a long-term investigation of the development of cardiovascular diseases from childhood to young adulthood. It is based on a panel design, consisting of repeated cross-sectional examinations, and participants have been followed longitudinally via their participation in these examinations over 30 years. Since 1973, this study has been following a community situated in Washington Parish (mainly including the city of Bogalusa) in southeastern Louisiana. The total population of this study area in 2000 was estimated to be 44,000 , a number which had remained almost unchanged since 1960 . The racial mix of the population was $67 \%$ white and $32 \%$ black (African American). From this entire population, the eligible population, which included all children and young adults up to the age of 26 years in 1977, comprised approximately 22,000 individuals.

Between 1973 and 1977, four cross-sectional surveys were conducted, with participation rates ranging between $80 \%$ and $93 \%$. Subjects ranged in age from 3 to 24 years. Between 1985 and 1996, three cross-sectional surveys were conducted when the subjects 
Table 1. Age, sex, and race data for subjects of this study

\begin{tabular}{|c|c|c|c|c|c|c|c|c|}
\hline & \multirow[b]{2}{*}{ Survey year } & \multirow[b]{2}{*}{$\mathrm{N}$} & \multicolumn{2}{|c|}{ Age (years) } & \multicolumn{2}{|c|}{ Male } & \multicolumn{2}{|c|}{ Female } \\
\hline & & & Mean (S.D) & Range & \%White & $\%$ Black & $\%$ White & $\%$ Black \\
\hline \multirow{4}{*}{$\begin{array}{l}\text { Baseline } \\
\text { population }\end{array}$} & $1973-74$ & 1834 & $10.5(2.9)$ & $4.0^{-15.4}$ & 30.0 & 14.2 & 36.4 & 19.4 \\
\hline & $1974-75$ & 597 & $11.2(3.2)$ & $6.0-16.0$ & 28.6 & 15.4 & 36.0 & 19.9 \\
\hline & $1976-76$ & 575 & $12.1(3.1)$ & $5.8-17.0$ & 28.3 & 15.7 & 35.5 & 20.5 \\
\hline & $1976-77$ & 1944 & $12.5(3.4)$ & $4.8-18.3$ & 30.2 & 14.0 & 36.7 & 19.1 \\
\hline \multirow{3}{*}{$\begin{array}{l}\text { Follow-up } \\
\text { population }\end{array}$} & $1985-86$ & 944 & $23.2(2.4)$ & $17.2-27.6$ & 29.8 & 10.3 & 43.1 & 16.8 \\
\hline & $1988^{-91}$ & 1794 & $25.8(3.2)$ & $19.2-32.6$ & 29.9 & 13.3 & 38.3 & 18.6 \\
\hline & $1995-96$ & 1016 & $31.8(3.7)$ & $24.0-37.9$ & 29.1 & 10.6 & 43.6 & 16.6 \\
\hline \multicolumn{2}{|c|}{ Total baseline surveys } & 2258 & $11.4(3.1)$ & $4.8^{-18.0}$ & 30.0 & 13.4 & 38.5 & 18.2 \\
\hline \multicolumn{2}{|c|}{ Total follow-up surveys } & 2258 & $26.3(3.6)$ & $17.8-37.9$ & 30.0 & 13.4 & 38.5 & 18.2 \\
\hline
\end{tabular}

were aged from 17 to 37 years. In this study, the earlier four surveys (mean subject age 11.3 years) were used for baseline data and the later three surveys (mean subject age 26.3 years) were used for follow-up data. By matching on ID number, only fasting subjects who participated in at least 1 of the baseline surveys (1973-1977) and 1 of the follow-up surveys (1985-1996) were eligible $(n=2317)$. Those with missing data about smoking status were excluded. The total number included in this analysis was 2258. The composition of this study population was $43 \%$ male and $68 \%$ white. Data from those subjects who participated in more than one survey for either the baseline or follow-up were summarized using the mean for each baseline and follow-up survey (Table 1). For categorical variables such as smoking status, the most recent follow-up survey data available were used.

\section{Smoking status}

Children in the third grade and higher were given questionnaires concerning smoking. Three categories were used to identify current smoking status from their most recent survey in the follow-up phase: (1) current smokers, who smoked at least 1 cigarette per week; (2) past smokers, who did not currently smoke but used to smoke at least 1 cigarette per week; and (3) nonsmokers, who never smoked or smoked less than 1 cigarette per week.

The number of cigarettes smoked per week or per day (both at present and in the past) was reported by all subjects including nonsmokers. In addition, the age when the subject started smoking was reported, as was the age when they stopped smoking (if applicable).

Smoking status was also defined by the number of pack-years ever smoked. For example, if a subject smoked 100 cigarettes per week for 5 years, pack-years were calculated as 100 multiplied by 5 (years) divided by 7 (days) and 20 (1 pack) to equal 3.57 pack-years. Subjects were categorized into 4 pack-year groups : 0-1 year, 1-3 years, 3-10 years, and > 10 years.

Because it is important to examine the number of years since quitting smoking when analyzing the beneficial effect of nonsmoking, the number of years a former smoker remained free of cigarettes was calculated from the difference between the age at the follow up survey and the age the individual stopped smoking. Former smokers were grouped accord- 
ing to the number of years since quitting smoking $(<1$ year, 1-3 years, 3-10 years, $>10$ years).

\section{General examination}

The variables used as major risk factors for CVD were systolic and diastolic blood pressure, TG, LDL-C, HDL-C, and body mass index (BMI). Trained examiners collected data under rigid protocols used consistently in each survey ${ }^{16)}$. After random assignment, examiners were sent to various on-site stations in Bogalusa to measure blood pressure, perform physical examinations, and collect blood samples and anthropometric data.

Blood pressures were measured with the subjects relaxed and sitting. Right arm systolic and diastolic blood pressures were measured in triplicate by each of two randomly assigned nurses. For the diastolic pressures, the $4^{\text {th }}$ Korotkoff phase was used for individuals aged less than 18 years, and the $5^{\text {th }}$ Korotkoff phase was used for individuals 18 years and older.

Subjects were instructed to fast for 12 hours prior to venipuncture; compliance was assessed by interview on the morning of the screening. Serum total cholesterol and TG concentrations were measured by enzymatic procedures using an Abbott VP analyzer (Abbott Laboratories, North Chicago, IL) ${ }^{17.18)}$. Lipoprotein cholesterol levels were measured by a combination of heparin-calcium precipitation and agar-agarose gel electrophoresis ${ }^{19)}$. The Heart Study laboratory maintains participation in the Lipid Standardization and Surveillance Program of the Centers for Disease Control and Prevention in Atlanta, GA. BMI was calculated by weight $(\mathrm{kg})$ divided by $\{\text { height }(\mathrm{m})\}^{2)}$. Weight and height were measured in duplicate and the averages were used for calculation.

\section{Statistical analysis}

All analyses were conducted using the statistical package SPSS ${ }^{20)}$. One-way ANOVA was conducted to measure the mean differences in changes in risk factor variables among the categories of smoking status ( 0 for non-smokers, 1 for past-smokers and 2 for current smokers), pack-years and number of years since quitting smoking. In addition, Pearson correlation coefficients between numbers of pack-years and the risk factor variables and other covariates were determined. Changes in risk factor variables were adjusted using multiple linear regressions separately for each sex and race group to control for the effects of baseline age, follow-up age, and respective baseline risk factor values. $P<.05$ was considered significant.

\section{Results}

\section{Smoking prevalence and behavior}

The age, sex and race distribution of the study population is shown in Table 2 . The mean age at baseline was 11.4 years, while the mean age at follow-up was 26.3 years.

Tables 2 to 4 show smoking status for the study population categorized in 3 different ways : by smoking status at the most recent follow-up survey, by pack-years of smoking and by years since quitting (stop-years). According to the smoking status at follow-up (Table 3 ), males were more likely to smoke than females (males $40.7 \%$ versus females $33.9 \%$; chisquare $=10.8, P<.01$ ), but there was no significant racial difference (whites $37.5 \%$ versus blacks $35.4 \%$; chi-square $=0.94, P>.05)$. Black males showed the highest $(44.0 \%)$ and black females the lowest $(29.0 \%)$ prevalence of current smoking. 
Table 2. Subject data categorized by smoking status at the most recent follow-up

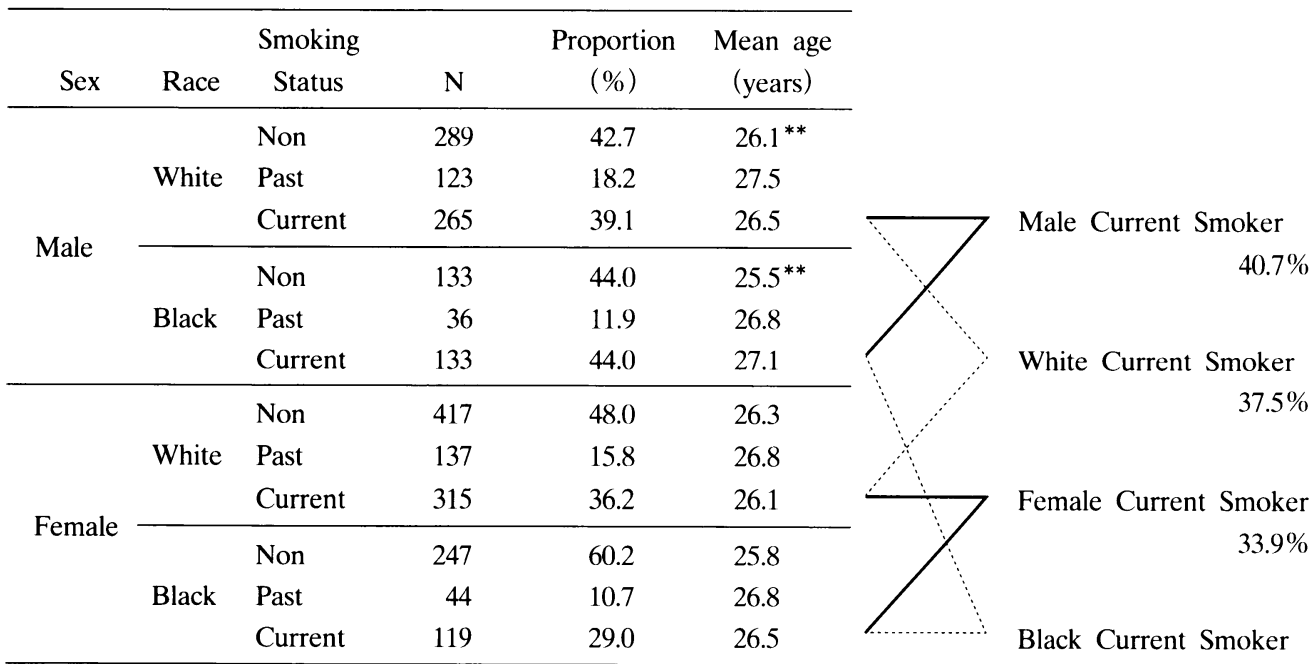

${ }^{* *} p<0.01$ for difference of mean age among smoking status.

Table 3. Subject data categorized by pack-years $(n=2258)$

\begin{tabular}{|c|c|c|c|c|c|c|c|c|}
\hline Pack-ye & & & 0 & $0<1$ & $1<3$ & $3<10$ & $10<$ & Total \\
\hline \multirow{4}{*}{ Male } & \multirow{2}{*}{ White } & $\mathrm{n}$ & 293 & 52 & 66 & 145 & 121 & 677 \\
\hline & & $\%$ & 43.3 & 7.7 & 9.7 & 21.4 & 17.9 & 100.0 \\
\hline & \multirow{2}{*}{ Black } & $\mathrm{n}$ & 144 & 35 & 38 & 52 & 33 & 302 \\
\hline & & $\%$ & 47.7 & 11.6 & 12.6 & 17.2 & 10.9 & 100.0 \\
\hline \multirow{4}{*}{ Female } & \multirow{2}{*}{ White } & $\mathrm{n}$ & 423 & 69 & 86 & 162 & 129 & 869 \\
\hline & & $\%$ & 48.7 & 7.9 & 9.9 & 18.6 & 14.8 & 100.0 \\
\hline & \multirow{2}{*}{ Black } & $\mathrm{n}$ & 257 & 52 & 34 & 48 & 19 & 410 \\
\hline & & $\%$ & 62.7 & 12.7 & 8.3 & 11.7 & 4.6 & 100.0 \\
\hline
\end{tabular}

Categorized by pack-years among persons reporting current smoking at the latest followup survey (Table 4), white smokers experienced more cumulative smoking than black smokers for both sexes. Mean pack-years were 7.5 years for whites and 3.2 years for blacks. Among males (both whites and blacks), nonsmokers were younger than past smokers or current smokers, but among females there were no significant differences.

Categorized by number of years since quitting smoking, more than $40 \%$ of past smokers of both sexes $(\mathrm{n}=333 ; 154$ male, 179 female $)$ had stopped smoking within the previous year. The mean number of pack-years smoked for those who had quit smoking was 5.03. Those who quit smoking more than 10 years previously tended to be older (mean age, 29.6 years versus 26.9 years for those who quit less than 10 years previously). There were no significant differences in mean pack-years smoked among those who had quit smoking $(P<.35)$. 
Table 4. Mean age and mean pack-years for subjects categorized by number of years since quitting smoking (stop-years $)(n=333)$

\begin{tabular}{|c|c|c|c|c|c|c|}
\hline SEX & $\begin{array}{l}\text { \# of years since } \\
\text { uitting smoking }\end{array}$ & $\mathrm{N}$ & $\%$ & $\begin{array}{l}\text { Age (years) } \\
\text { Mean Age }\end{array}$ & $\begin{array}{c}\text { Pack-years } \\
\text { Mean (S.D.) }\end{array}$ & $\begin{array}{c}\text { sig. } \\
\text { ANOVA }^{\dagger}\end{array}$ \\
\hline \multirow{5}{*}{ Male } & within 1 year & 68 & 44.2 & 27.8 & $6.0(6.3)$ & 0.427 \\
\hline & 1 to 3 years & 42 & 27.3 & $25.8^{* *}$ & $5.6(7.5)$ & \\
\hline & 3 to 10 years & 33 & 21.4 & 27.9 & $3.8(3.7)$ & \\
\hline & over 10 years & 11 & 7.1 & $29.3^{* *}$ & $5.2(5.9)$ & \\
\hline & Total & 154 & 100.0 & 27.4 & $5.4(6.2)$ & \\
\hline \multirow{5}{*}{ Female } & within 1 year & 83 & 46.4 & 27.6 & $5.4(7.9)$ & 0.734 \\
\hline & 1 to 3 years & 44 & 24.6 & $24.5^{* *}$ & $4.3(5.9)$ & \\
\hline & 3 to 10 years & 45 & 25.1 & 27.0 & $4.0(7.8)$ & \\
\hline & over 10 years & 7 & 3.9 & $30.0^{* *}$ & $4.4(7.0)$ & \\
\hline & Total & 179 & 100.0 & 26.8 & $4.7(7.3)$ & \\
\hline
\end{tabular}

${ }^{* *} p<0.01$ for difference of mean age among categories

${ }^{+}$sig. ANOVA examined the difference of mean pack-years among categories

Table 5. Age and risk factor variables from baseline and follow-up surveys

\begin{tabular}{|c|c|c|c|c|}
\hline \multirow[b]{3}{*}{$\mathrm{N}$} & \multicolumn{2}{|c|}{ Male } & \multicolumn{2}{|c|}{ Female } \\
\hline & White & Black & White & Black \\
\hline & 677 & 302 & 869 & 410 \\
\hline Age (baseline) & 11.6 & 11.7 & 11.2 & 11.2 \\
\hline Age (change) & 14.9 & 14.7 & 15.1 & 14.9 \\
\hline Sys. BP (baseline) (mmHg) & 102.6 & 102.5 & 100.6 & 101.1 \\
\hline Sys. BP (change) & 11.3 & 13.6 & 6.6 & 9.6 \\
\hline Dias. BP (baseline) (mmHg) & 62.7 & 63.4 & 62.7 & 63.3 \\
\hline Dias. BP (change) & 11.0 & 10.8 & 7.3 & 7.9 \\
\hline TG (baseline) $(\mathrm{mg} / \mathrm{dl})$ & 70.3 & 60.0 & 74.6 & 62.2 \\
\hline TG (change) & 55.0 & 48.6 & 40.2 & 18.8 \\
\hline HDL-C (baseline) (mg/dl) & 61.6 & 68.4 & 61.7 & 68.6 \\
\hline HDL-C (change) & -18.5 & -12.7 & -10.6 & -11.2 \\
\hline LDL-C (baseline) (mg/dl) & 84.2 & 84.0 & 87.8 & 88.9 \\
\hline LDL-C (change) & 36.3 & 26.8 & 28.2 & 23.3 \\
\hline BMI (baseline) $\left(\mathrm{kg} / \mathrm{m}^{2}\right)$ & 18.8 & 18.4 & 18.2 & 19.0 \\
\hline BMI (change) & 7.3 & 7.1 & 6.1 & 8.0 \\
\hline
\end{tabular}

For all variables except age (change) and Sys.BP (baseline), there were significant differences between the 4 sex-race groups as determined by ANOVA. $(P<.01)$ 
Table 6. Adjusted changes in risk factor variables between baseline and follow-up surveys categorized by smoking status at the most recent follow-up

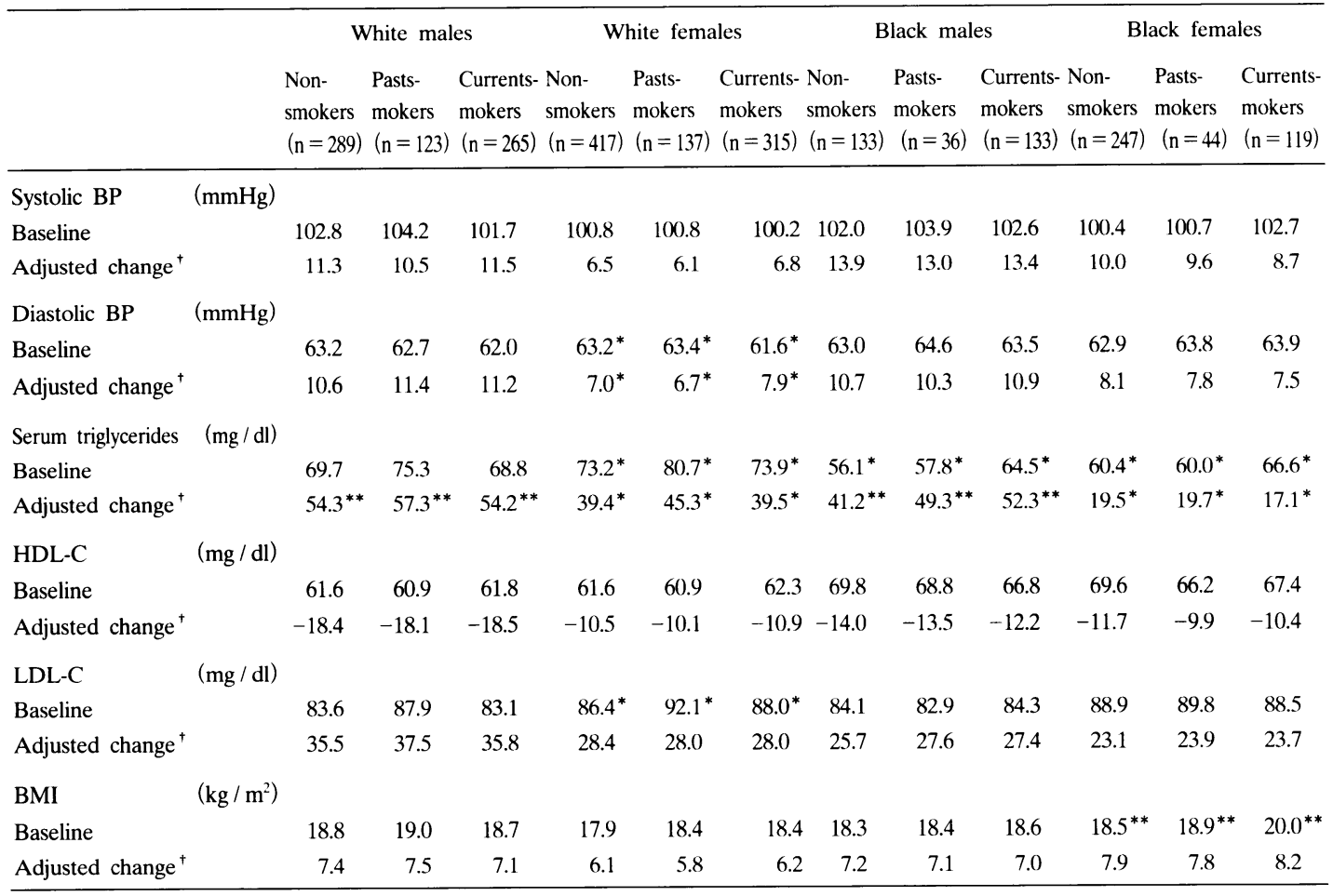

$\dagger$ Adjusted changes were calculated by using multiple linear regression to control for the effects of respective baseline data, baseline age and follow-up age.

${ }^{*} p<0.05$ for difference among three smoking status.

${ }^{* *} p<0.01$ for difference among three smoking status.

Table 5 shows the mean age and risk factor variables at baseline and changes at followup for each sex-race category. There were no significant differences in age at baseline or follow-up among the 4 sex-race groups. However, there were significant differences between the groups in the risk factor variables at baseline and in the changes at follow-up, with the exception of the baseline data for systolic blood pressure.

\section{Relationship of smoking to the risk factor variables}

Adjusted changes in risk factor variables among subjects categorized by smoking status at follow-up are shown in Table 6. There were no significant differences in change of systolic blood pressure related to current smoking status. White females who were current smokers showed the largest increase in diastolic blood pressure $(P<.05)$. For TG, white males and females who were categorized as past smokers had the largest significant increases compared to nonsmokers and current smokers (males: $57.3 \mathrm{mg} / \mathrm{dL}$ for past-smokers versus $54.3 \mathrm{mg} /$ $\mathrm{dL}$ for nonsmokers and current smokers, $P<.01$; females : $45.3 \mathrm{mg} / \mathrm{dL}$ for past-smokers versus $39.5 \mathrm{mg} / \mathrm{dL}$ for nonsmokers and current smokers, $P<.01$ ). However, for black males, current smokers had the largest increases in TG $(P<.01)$, while for black females, current smokers had the smallest estimated increases $(P<.05)$. For HDL-C, LDL-C, and 

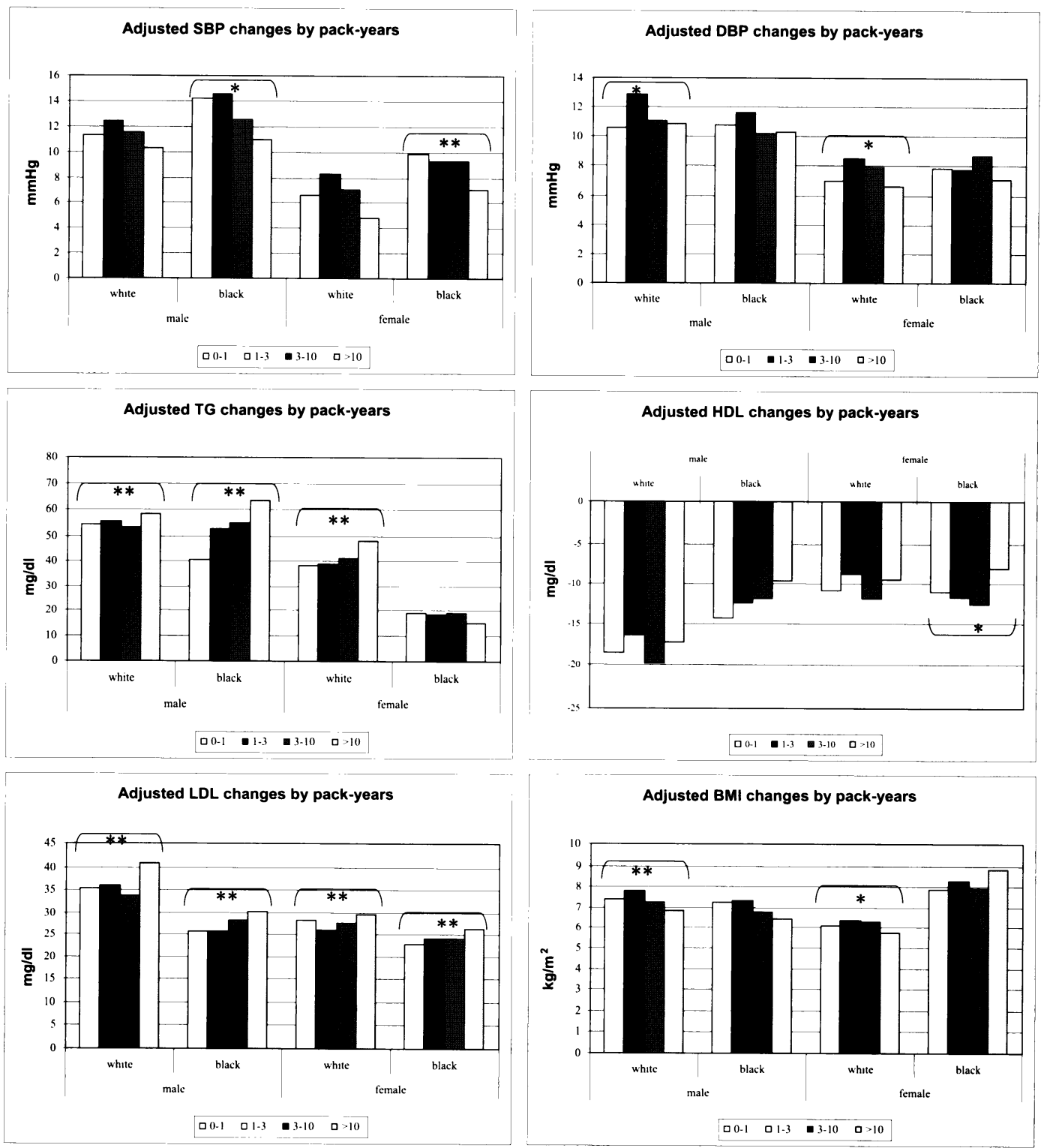

Fig. 1. Adjusted risk factor variable changes among the 4 sex-race groups categorized by pack-years Categorized into 4 pack-year groups: $0-1$ year, 1-3 years, 3-10 years. $>10$ years.

Adjusted for the respective baseline data, baseline age and follow-up age.

${ }^{*} P<.05$ for differences between the 4 pack-year groups.

${ }^{* *} P<.01$ for differences between the 4 pack-year groups.

BMI, no significant differences were found when comparing category of smoking status with the adjusted change between baseline and follow-up.

Adjusted changes in risk factor variables among subjects categorized by pack-years are shown in Fig. 1. For systolic blood pressure, in all the sex-race groups the smallest increase between baseline and follow-up was for those with more than 10 pack-years of 

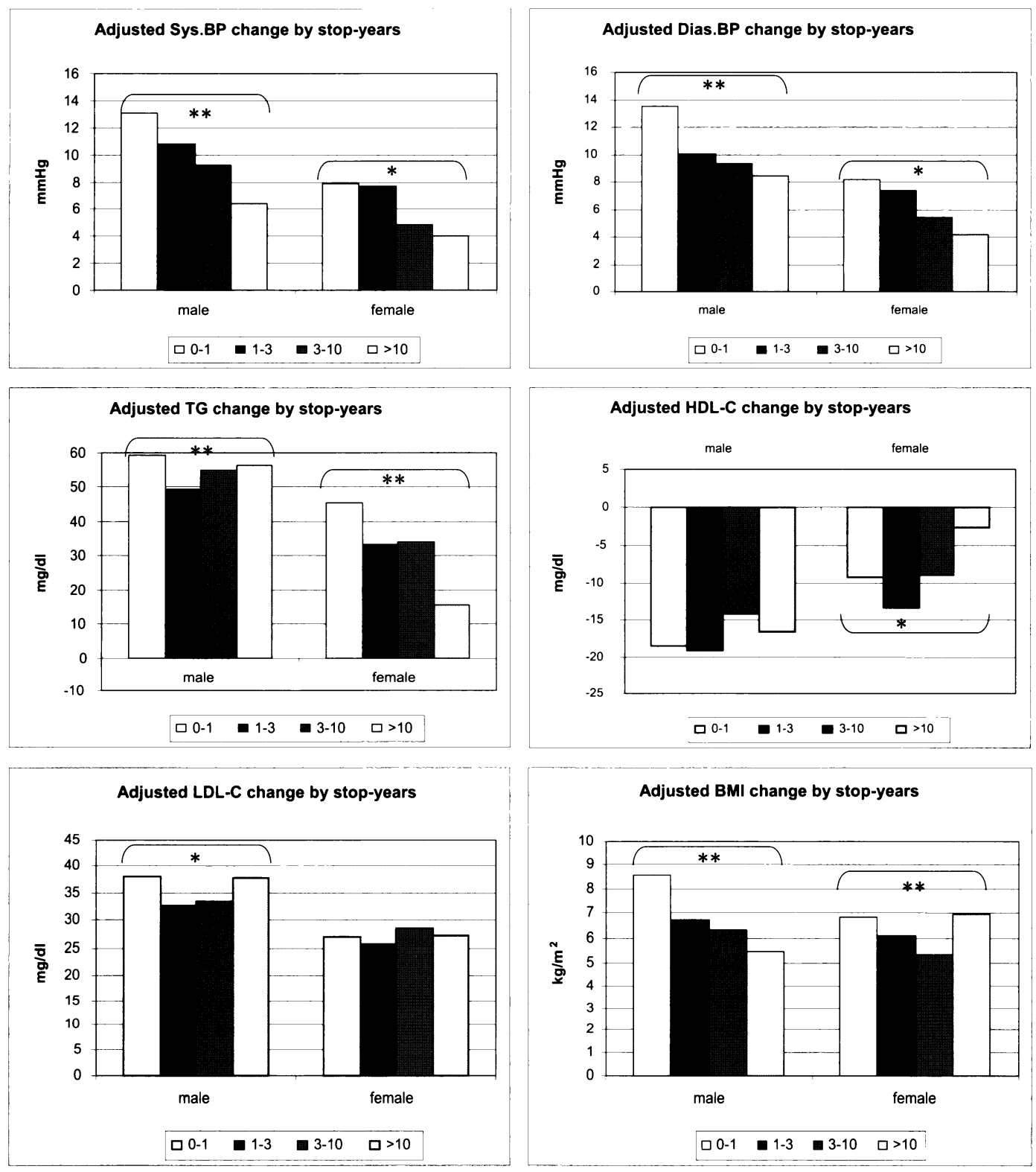

Fig. 2. Adjusted risk factor variable changes categorized by years since quitting smoking (stop-years) Categorized into 4 groups by number of years since quitting smoking: $0^{-1}$ year, 1-3 years, 3-10 years, $>10$ years.

Adjusted for the respective baseline data, baseline age and follow-up age.

${ }^{*} P<.05$ for differences between the 4 pack-years groups.

${ }^{* *} P<.01$ for differences between the 4 pack-years groups.

smoking (significant for black males, $P<.05$; black females, $P<.01$ ). There was no consistent pattern for diastolic blood pressure. For TG, both black and white males and white females had larger increases between baseline and follow-up in those with greater 
than 10 pack-years of smoking $(P<.01)$. Black males with more than 10 pack-years showed the largest increase in TG levels compared to never-smokers (a $21.3 \mathrm{mg} / \mathrm{dL}$ greater increase). Black males who smoked the largest number of pack-years had the smallest HDL-C decreases, although this was not significant, and this tendency was not seen in other sex-race groups. For LDL-C, those with more than 10 pack-years of smoking had the largest adjusted increases in all 4 sex-race groups $(P<.01)$. BMI showed the largest change between baseline and follow-up for those white males and white females who smoked between 1 and 3 pack-years (white males, $P<.01$; white females, $P<.05$ ).

Fig. 2 shows adjusted changes in risk factor variables between baseline and follow-up surveys categorized by number of years since quitting smoking (stop-years). Because of the small sample sizes, the analysis was divided into groups by sex. Those with the longest number of years since quitting showed significantly smaller changes of systolic and diastolic blood pressure in both sexes (males, $P<.01$; females, $P<.05$ ). For TG, those who quit within the previous year had larger changes from baseline compared to those who quit more than 10 years previously (males and females, $P<.01$ ). Females who quit between 1 and 3 years previously had the greatest decrease in HDL-C and those who quit more than 10 years previously had the smallest decrease $(P<.05)$. Males who quit smoking most recently had the greatest increase in LDL-C $(P<.05)$. For BMI changes between baseline and follow-up, males showed the largest gain in those who quit smoking within the previous year $(P<.05)$. In females, those who quit more than 10 years previously showed the largest gain in BMI $(P<.01)$.

\section{Discussion}

In this study, the association between changes in CVD risk factors and smoking as measured by current smoking status, pack-years (cumulative volume) and years since quitting smoking was examined in a stable population in Bogalusa over a 15-year time period. At baseline (mean age-11.4 years old) only $6.65 \%$ categorized themselves as being a smoker, but by the time of the last follow-up survey (mean age-26.3 years old) $51.9 \%$ were smokers (including $15.1 \%$ past smokers).

When subjects were compared according to current smoking status, the only significant difference seen across all sex-race groups was in the adjusted change in TG levels. It was expected that nonsmokers should have the lowest adjusted increase in TG but this was only observed for white females and black males. When the relationship between the risk factor changes and cumulative smoking (pack-years) was examined, TG and LDL-C levels changed as expected with the highest changes seen in those who smoked the longest (except for TG in black females). Blood pressure and HDL-C did not show consistent or expected patterns. The adjusted change in BMI showed the largest change for those who had smoked the second least number of pack-years (1-3 years).

When the relationship between risk factor changes and years since quitting smoking was examined, blood pressure and TG followed the expected pattern, with those who had quit most recently having the highest levels. With HDL-C, the longer number of years of not smoking showed the lowest or second-lowest amount of adjusted change. For BMI, those who quit most recently had the largest change.

When the relationship between changes in risk factors and the different measures of smoking status was examined, it was found that pack-years was able to distinguish the most 
significant changes from baseline to follow-up. Years since quitting smoking also showed a number of significant changes from baseline, but because of the limited number of individuals, racial differences could not be examined. Current smoking status did not capture the cumulative effects of smoking on the risk factors and was not able to distinguish many changes from baseline.

Critchley and Capewell ${ }^{21)}$ reported a $36 \%$ reduction in crude relative risk of mortality for those who quit smoking compared with those who continued to smoke. The risk reduction associated with quitting smoking seems consistent regardless of differences between studies in terms of index cardiac events, age, sex, country, and time period. Our data for the number of years since quitting smoking therefore has the potential to predict future CVD morbidity and mortality in the population of young adults that we studied.

Our results did not show significant changes in blood pressure associated with current smoking status, and an inverse trend was observed with pack-years. It has been shown that smoking raises blood pressure acutely, with a return to normal 20 minutes after smoking is stopped ${ }^{11}$. Several previous studies have shown a slightly lower blood pressure among smokers compared to nonsmokers ${ }^{12,22)}$. The results of this study are therefore consistent with those previous studies, and show an appropriate dose-response gradient.

In the population we studied, those who quit smoking tended to have lower increases in both systolic and diastolic blood pressure, with a clear relationship to the number of years since quitting. There were no significant differences in the means of the pack-years among the 4 stop-year groups. However, those quitting over 10 years ago were older $(P<.01)$ and age is also related to higher blood pressure. Despite the expected contrary effect of age, our results showed that longer the time since quitting smoking, the more favorable the change. Our findings also suggest that some of the adverse effects of smoking on CVD may be reversible in young adulthood, while they may be irreversible in older age groups $(>45 \text { years })^{15)}$.

The association between current smoking status and TG change was inconsistent among the sex-race groups. However, when categorized by pack-years, there was a tendency for increases in cumulative smoking to correspond with larger increases in TG levels (except in black females). On the other hand, when categorized by number of years since quitting smoking, females who had stopped smoking for the longest period showed the smallest mean increase in TG. This effect was not seen in males.

The relationship between current smoking status and changes in serum lipoprotein fractions was not clear nor consistent among the 4 sex-race groups. When the population was divided into cumulative smoking groups (pack-years), clearer associations were seen. The heavier smokers tended to have the larger increases in TG and LDL-C. The meta-analysis study conducted by Maeda et $a^{23}$ ) showed that cigarette smoking cessation increases serum levels of HDL-C, but does not significantly decrease levels of total cholesterol, LDL-C, or TG. In the present study, when smoking was categorized by stop-years, significantly smaller changes in TG were found in the female group with the greatest number of stop-years, but not in the corresponding male group. For HDL-C and LDL-C, there was no clear association with the number of years since quitting.

Smoking, oral contraceptive use, and alcohol consumption are 3 major behaviors that influence CVD risk factors ${ }^{24,25)}$. In this study, oral contraceptive use and alcohol consumption were not examined, but some previous reports from the Bogalusa Heart Study have 
studied the effects of alcohol or oral contraceptive use, and the interaction between them and smoking on serum lipoproteins ${ }^{22,26,27)}$.

It is often observed that after individuals stop smoking, they tend to seek an alternative addiction such as alcohol or food ${ }^{28.29)}$. The association between smoking and weight has been well studied, and an average long-term weight gain of 6 to $8 \mathrm{lb}(2.7-3.6 \mathrm{~kg})$ has been found for individuals who quit smoking ${ }^{28.29)}$. In the present study, with subjects followed from childhood to young adulthood, the crude increases in BMI were significantly different depending upon current smoking status. The largest gain was among nonsmokers, and the second largest among past smokers. Current smokers had the smallest gain in BMI. However, these differences were not clear in the adjusted changes. When categorized by stop-years, the largest gain in adjusted BMI occurred for those males who had quit within the previous year. However, for females, those who quit more than 10 years ago showed the largest gain in BMI. Possible reasons for the differences between males and females include oral contraceptive use, alcohol or diet habitus.

Analysis of the association between lipid levels and BMI in a study by Devroey et al ${ }^{30}$ ) showed that obesity was correlated with low HDL-C and high TG. In the Japanese population the influence of life-style factors such as cigarette smoking and alcohol consumption on serum lipid parameters are mostly expressed as a function of BMI in younger men, but are independent of BMI in older men ${ }^{31)}$. Smokers also have a less healthy diet than nonsmokers ${ }^{32)}$. Programs that help individuals stop smoking should therefore include information on how to improve diet.

Finally, quitting smoking at an earlier age is associated with greater reductions in mortality of all causes ${ }^{33)}$. In the present study, those who had stopped smoking for the longest time showed the most beneficial compensations of their CVD risk factor variables.

\section{Conclusion}

The methods used to measure smoking status influenced the results obtained. Measurement of cumulative smoking (pack-years) showed the most significant relationships with risk factor variables. The number of years since quitting smoking also showed the appropriate inverse relationship to many of these variables. This study provides further evidence that between childhood and young adulthood, smoking promotes unfavorable changes in risk factor variables for CVD such as blood pressure, serum lipoproteins, and BMI. Our findings also suggest that quitting smoking for a long period of time can compensate for the unfavorable changes that occur in those risk factors in young adulthood.

\footnotetext{
Abbreviations BMI, body mass index; BP, blood pressure : CVD, Cardiovascular disease; HDL-C, high density lipoprotein cholesterol ; LDL-C, low density lipoprotein cholesterol ; TG, serum triglycerides

\section{Acknowledgements}

This study was supported by the National Institute of Child and Human Health, HD43820-01; the National Heart, Lung and Blood Institute, HL38844; and the National Institute of Aging, AG16592.
}

\section{References}

1) Annex Table 7 Selected population attributable fractions by risk factor, sex and level of development (\% DALYs for each cause). In: The World Health Report 2002: Reducing Risks, Promoting Healthy Life, World 
Health Organization, Geneva, pp 220-221 (2002)

2) Doll $\mathrm{R}$ and Peto $\mathrm{R}$ : Mortality in relation to smoking: 20 years' observations on male British doctors. $\mathrm{Br} \mathrm{Med}$ J 2 : 1525-1536 (1976)

3) Annex Table 11 Attributable mortality by risk factor, sex and mortality stratum in WHO Regions. In: The World Health Report 2002: Reducing Risks, Promoting Healthy Life, World Health Organizorlian, Geneva, pp 226-227 (2002)

4) Department of Health and Human Services, The Centers for Disease Control and Prevention (CDC), (online), available from $<$ http: // www.cdc.gov / $>$, (accessed 2005-7-1).

5) Hammond EC: Smoking in relation to mortality and morbidity. Findings in first thirty-four months of followup in prospective study started in 1959. J Natl Cancer Inst $32: 1161-1188$ (1964)

6) Kannel WB: Update on the role of cigarette smoking in coronary artery disease. Am Heart $J$ 101 : 319-328 (1981)

7) Reducing Tobacco Use: A Report of the Surgeon General, U.S, Department of Health and Human Services, Centers for Disease Control and Prevention, National Center for Chronic Disease Prevention and Health Promotion, Office on Smoking and Health, Atlanta (2000)

8) Centers for Disease Control and Prevention (CDC): Annual smoking-attributable mortality, years of potential life lost, and economic costs - United States, 1995-1999. Morb Mortal Wkly Rep 51 : 300-303, April 12 (2002)

9) Miniño AM, Arias E, Kochanek KD, Murphy SL and Smith BL: Deaths: final data for 2000. National Vital Statistics Reports 2002 50(15) : 1-120 (2002)

10) Mokdad AH, Marks JS, Stroup DF and Gerberding JL: Actual causes of death in the United States, 2000. JAMA 291 : 1238-1246 (2004)

11) The Health Benefits of Smoking Cessation: A Report of the Surgeon General. U.S Department of Health and Human Services, Public Health Service, Centers for Disease Control, Center for Chronic Disease Prevention and Health Premotion, Office on Smoking and Health, Rockville, Md. (1990) (DHHS publication; no. (CDC) 90-8416)

12) Garrison RJ, Kannel WB, Feinleib M, Castelli WP. McNamara PM and Padgett SJ : Cigarette smoking and HDL cholesterol: The Framingham offspring Study. Atherosclerosis 30 : 17-25 (1978)

13) Weintraub WS, Klein LW, Seelaus PA, Agarwal JB and Helfant RH: Importance of total life consumption of cigarettes as a risk factor for coronary artery disease. Am J Cardiol $55: 669-672$ (1985)

14) Tell GS, Polak JF, Ward BJ, Kittner SJ, Savage PJ and Robbins J : Relation of smoking with carotid artery wall thickness and stenosis in older adults. The Cardiovascular Health Study. The Cardiovascular Health Study (CHS) Collaborative Research Group. Circulation 90 : 2905-2908 (1994)

15) Howard G, Wagenknecht LE, Burke GL, Diez-Roux A, Evans GW, McGovern P, Nieto FJ and Tell GS: Cigarette smoking and progression of atherosclerosis: The Atherosclerosis Risk in Communities (ARIC) Study. JAMA 279 : 119-124 (1998)

16) Cardiovascular Risk Factors in Children: the Early Natural History of Atherosclerosis and Essential Hypertension, Berenson GS, McMahan CA, Voors AW, Webber LS, Srinivasan SR, Frank GC, Foster TA and Bland CV (Eds) : Oxford University Press, New York (1980)

17) Allain CC, Poon LS, Chan CSG, Richmond W and Fu PC: Enzymatic determination of total serum cholesterol. Clin Chem $20: 470-475$ (1974)

18) Bucolo $\mathrm{G}$ and David $\mathrm{H}$ : Quantitative determination of serum triglycerides by the use of enzymes. Clin Chem 19:476-482 (1973)

19) Srinivasan SR and Berenson GS: Serum lipoprotein in children and methods for study. In: Lipoprotein Methodology and Human Studies, Lewis LA (Eds), CRC Press, Boca Raton, FLa., pp 185-204 (1983) (CRC handbook of electrophoresis; V.3)

20) SPSS Inc: SPSS version 10.0.7, SPSS Inc, Chicago, 1989-1999

21) Critchley $\mathbf{J}$ and Capewell $\mathbf{S}$ : Smoking cessation for the secondary prevention of coronary heart disease. Cochrane Database Syst Rev (1): CD003041 (2004)

22) Croft JB, Freedman DS, Cresanta JL, Srinivasan SR, Burke GL, Hunter SM, Webber LS, Smoak CG and Berenson GS : Adverse influences of alcohol, tobacco, and oral contraceptive use on cardiovascular risk factors during transition to adulthood. Am J Epidemiol 126:202-213 (1987)

23) Maeda $K$, Noguchi $Y$ and Fukui $T$ : The effects of cessation from cigarette smoking on the lipid and lipoprotein profiles: a meta-analysis. Prev Med $37: 283-290$ (2003)

24) Kaplan NM and Stamler J : Prevention of Coronary Heart Disease: Practical Management of the Risk Factors. Saunders, Philadelphia (1983)

25) Wahl P, Walden C, Knopp R, Hoover J, Wallace R, Heiss G and Rifkrnd B : Effect of estrogen/progestin potency on lipid / lipoprotein cholesterol. N Engl J Med 308: 862-867 (1983)

26) Webber LS, Hunter SM, Baugh JG, Srinivasan SR, Sklov MC and Berenson GS: The interaction of cigarette 
smoking, oral contraceptive use, and cardiovascular risk factor variables in children: The Bogalusa Heart Study. Am J Public Health 72 : 266-274 (1982)

27) Freedman DS, Srinivasan SR, Shear CL, Hunter SM, Croft JB, Webber LS and Berenson GS: Cigarette smoking initiation and longitudinal changes in serum lipids and lipoproteins in early adulthood: The Bogalusa heart study. Am J Epidemiol $124: 207-219$ (1986)

28) West R. Tobacco withdrawal symptoms. St. George's Hospital Medical School, London (1996)

29) Stamford BA, Matter S, Fell RD and Papanek P: Effects of smoking cessation on weight gain, metabolic rate, caloric consumption, and blood lipids. Am J Clin Nutr 43 : 486-494 (1986)

30) Devroey D, DeSwaef N, Coigniez P, Vandevoorde J, Kartounian J and Betz W: Correlations between lipid levels and age, gender, glycemia, obesity, diabetes, and smoking. Endocr Res 30:83-89 (2004)

31) Yamamoto A, Temba H, Horibe H, Mabuchi H, Saito $Y$, Matsuzawa $Y$, Kita T, Nakamura $H$ and Research Group on Serum Lipid Survey 1990 in Japan: Life style and cardiovascular risk factors in the Japanese population- from an epidemiological survey on serum lipid levels in Japan 1990 Part 1: Influence of life style and excess body weight on HDL-cholesterol and other lipid parameters in men. $J$ Atheroscler Thromb 10:165-175 (2003)

32) Dyer AR, Elliott P, Stamler J, Chan Q, Ueshima H, Zhou BF and INTERMAP Research Group: Dietary intake in male and female smokers, ex-smokers, and never smokers: The INTERMAP study. Journal of Human Hypertension $17: 641-654$ (2003)

33) Doll R, Peto R, Boreham $\mathrm{J}$ and Sutherland $\mathrm{I}$ : Mortality in relation to smoking: 50 years' observations on male British doctors. BMJ $328: 1519$ (2004)

[Received January 10, 2006 : Accepted February 10, 2006] 